
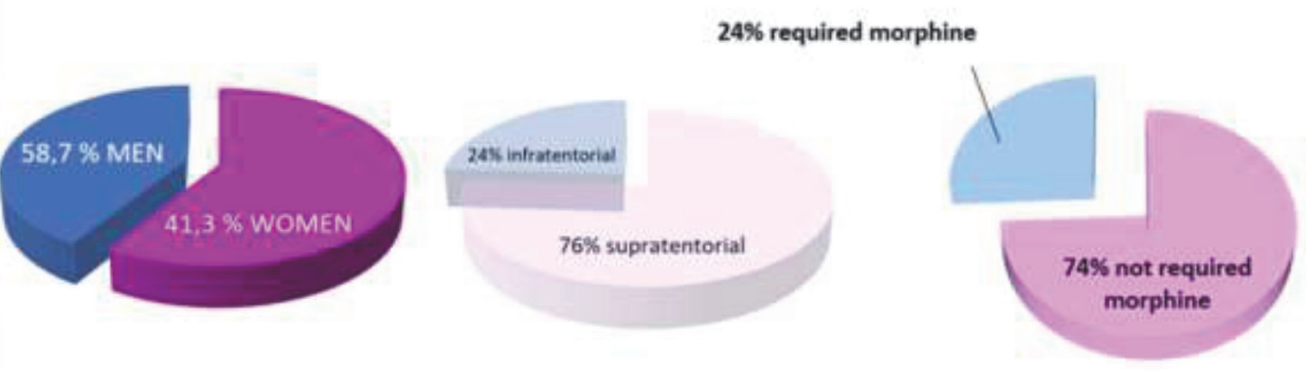

Abstract 223 Figure 1 Average pain scores

We hypothesize that adequate post-operative pain management using continuous supra-inguinal fascia illiaca catheter (SIFC) will reduce incidence of dementia in hip fracture patients.

Methods We evaluated a new protocol for post-operative pain management in hip fracture surgeries in a tertiary referral, Level 1 equivalent trauma centre. Upon theatre arrival, pericapsular injection $10 \mathrm{ml}$ lignocaine $2 \%$ followed by threading of supra-inguinal fascia illiaca catheter (SIFC) under ultrasound guidance. Continuous bupivacaine $0.2 \%$ infusion was started postoperatively at rate of $5 \mathrm{ml} / \mathrm{hr}$ for $48 \mathrm{hrs}$.

Pain was assessed $10 \mathrm{~min}$ after peri-capsular injection during positioning for spinal anaesthesia (lateral position) on a scale $0-10$. and every 12 hrs postoperatively for 48 hrs. Delirium was assessed upon theatre admission and every 12 hrs for 48 hrs using CAM ICU.

Results Twenty patients who had hip fracture surgery were successfully followed up, two patients were excluded because catheter was slipped in the first 24 hours. The incidence of new onset delirium was found to be $10 \%(2 / 20)$, there was $58 \%$ reduction in the incidence of delirium among traumatic hip fracture patients when compared to the literature $(24 \%){ }^{(1,2)}$ The Morphine Milligrams Equivalent (MME) in the first $24 \mathrm{hrs}$ are $34 \pm 27$, while in the second $24 \mathrm{hrs} 32$ \pm 25 .

Conclusions Implementation of supra-inguinal fascia illiaca continuous block could reduce incidence of delirium in hip fracture population by up to $58 \%$.

\section{SAFETY AND EFFICACY OF SUFENTANIL SUBLINGUAL TABLET SYSTEM (SSTS) FOR POSTOPERATIVE PAIN (POP) RELIEF AFTER OFF-PUMP CORONARY ARTERY BYPASS SURGERY (OPCABG)}

A Laudani*, R Gammaldi. AOU San Giovanni di Dio e Ruggi d'Aragona, Salerno, Italy

10.1136/rapm-2021-ESRA.224

Background and Aims POP in cardiac surgery is often overlooked, being moderate to severe in up to $75 \%$ of patients. Moreover, 35\% of patients report persistent thoracic pain one year post surgery. SSTS is a handheld PCA device that delivers a fixed dose of $15 \mathrm{mcg}$ sufentanil tablets on a PRN basis. We evaluated clinical application of SSTS for POP relief after OPCABG, assessing effectiveness, safety, and patient satisfaction.

Methods Observational case series on 20 patients who underwent OPCABG. POP was managed by the exclusive use of SSTS. Prior to the end of surgery, paracetamol $1 \mathrm{~g}$, sufentanil
0.15-0.2 $\mathrm{mcg} / \mathrm{kg}$, ondansetron $4 \mathrm{mg}$ were administered. Efficacy was assessed by patient reports of pain intensity on numerical rating scale (NRS). Safety assessments included vital signs. Patient satisfaction was assessed via the Patient Global Assessment (PGA) of method of pain control, with 'success' defined as the proportion of patients responding 'good' or 'excellent'.

Results Average patient age was 61 years, BMI was 26.6. Mean number of doses was 20 (range 8-52) over 72 hours, with inter-dosing intervals of 159 minutes. Median NRS was 1 (range 0-4) at rest, and 2 (range 0-6) during movement. No desaturation $(\mathrm{SpO} 2<92 \%)$ was found. PGA scores showed a success rate of $91 \%$. Neither harvesting nor cannulation of the radial artery of the dominant limb limited the use of SSTS.

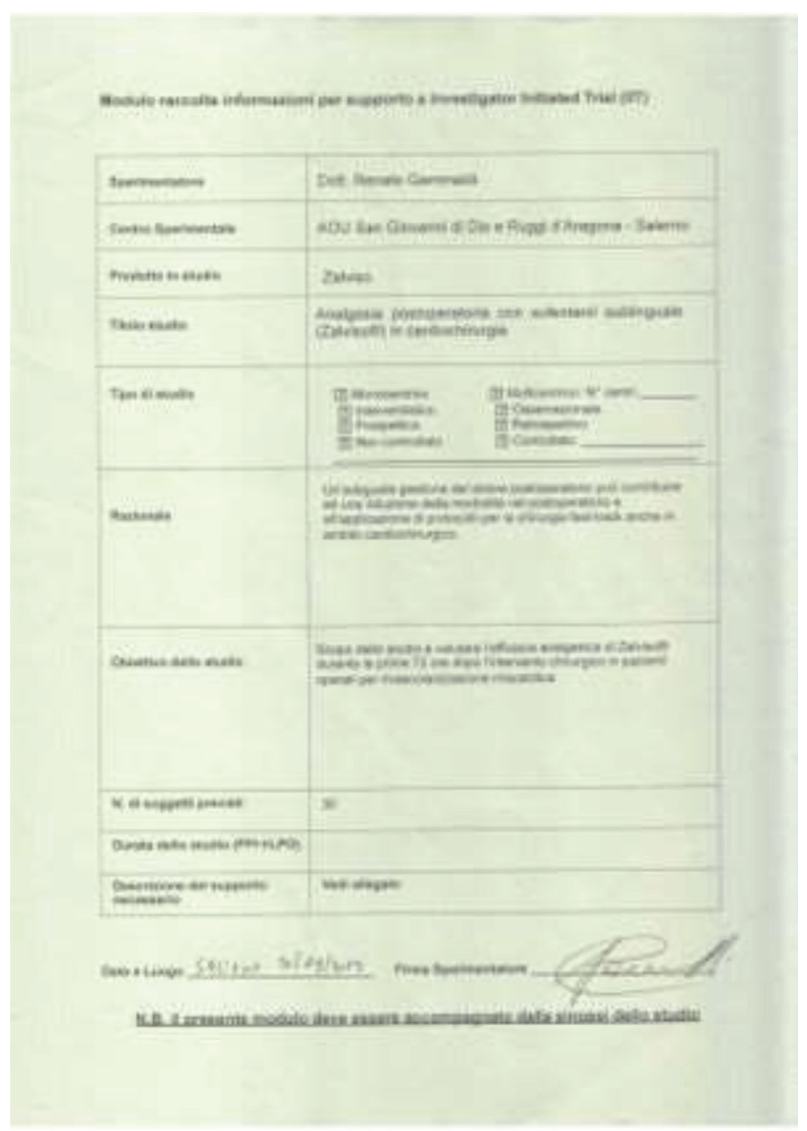

Abstract 224 Figure 1 
Conclusions SSTS has proved an effective and safe device for POP relief after OPCABG. To the best of our knowledge, this is the first report of SSTS use in OPCABG patients.

\section{CONTINUOUS ULTRASOUND-GUIDED RECTUS-SHEATH BLOCK FOR MIDLINE LAPAROTOMY: AN EFFECTIVE POSTOPERATIVE PAIN MANAGEMENT TECHNIQUE - CASE SERIES}

C Pinto, F Matias*. Centro Hospitalar e Universitário de Coimbra, Coimbra, Portugal

\subsection{6/rapm-2021-ESRA.225}

Background and Aims Epidural analgesia, though the gold standard of post-operative pain management for laparotomies, is associated with limitations and is contraindicated in many patients. Continuous rectus sheath (RS) block can provide alternative analgesia after midline laparotomy.

Methods We report 5 cases in which continuous local anesthetic infusion through ultrasound-guided (USG) RS catheters showed effective postoperative pain management for midline laparotomy.The multimodal approach for postoperative analgesia for all 5 patients was: paracetamol $1 \mathrm{~g} 8 / 8 \mathrm{~h}$, ketorolac 30 $\mathrm{mg} 12 / 12 \mathrm{~h}$ or metamizole $2 \mathrm{~g} 8 / 8 \mathrm{~h}$ and ropivacaine $0,2 \%$ through DIB system $(5,2 \mathrm{ml} / \mathrm{h})$ through a bilateral percutaneous USG RS catheter during $72 \mathrm{~h}$. Tramadol $100 \mathrm{mg}$ as rescue analgesia.

Results Patient's pain intensity assessed by numerical rating scale during first $72 \mathrm{~h}$ and the number of times necessary to administer rescue analgesia was also recorded (see table 1). Conclusions With this case series, the authors showed that bilateral percutaneous USG RS catheter as part of a

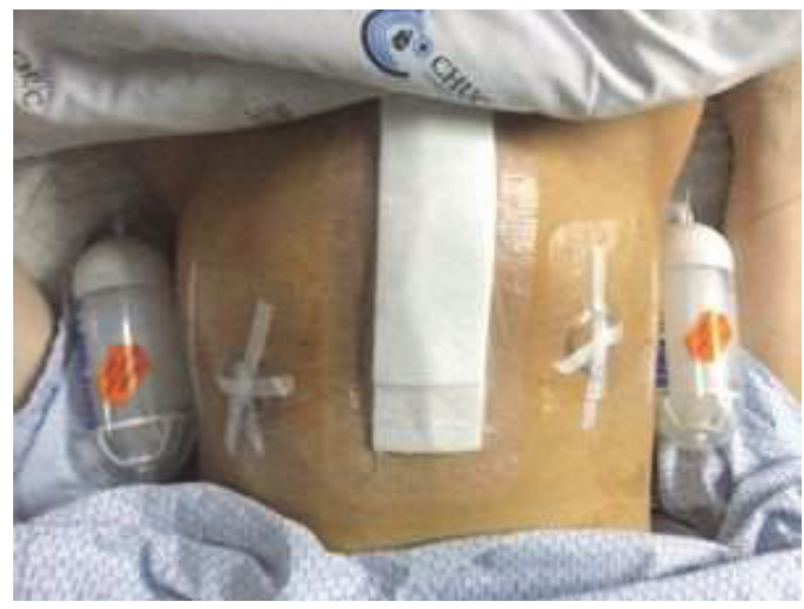

Abstract 225 Figure 1

\section{Abstract 225 Table 1}

\begin{tabular}{|c|c|c|c|c|c|}
\hline $\begin{array}{c}\text { Pain Scores (0- } \\
\text { 10) }\end{array}$ & Care 1 & Care 2 & $\cos 3$ & ches 4 & Cones \\
\hline Worst pain & 4 & 5 & 3 & 3 & 4 \\
\hline Medlum pain & 1 & 1 & 1 & 1 & 1 \\
\hline $\begin{array}{l}\text { Rescuse } \\
\text { andergesis }\end{array}$ & & & & & \\
\hline $\begin{array}{c}\text { Administration } \\
\text { times }\end{array}$ & 0 & 1 & 0 & 0 & 2 \\
\hline
\end{tabular}

multimodal analgesia regime seems to provided effective postoperative analgesia for midline laparotomy with minimal or no opioid needs.

\section{USG GUIDED TAP BLOCK FOR POST OPERATIVE ANALGESIA IN CHILDREN WITH COVID INFECTION UNDERGOING LAPAROTOMY}

A Praniarda*. Brawijaya University, Malang, Indonesia

\subsection{6/rapm-2021-ESRA.226}

Background and Aims The COVID-19 infection that broke out in the past year had a major impact on pediatric surgery. The urgency of surgical management and asymptomatic symptom in pediatric patients to the risk of multiorgan inflammatory syndrome makes anesthesiologists consider safe and optimal anesthetic techniques.

Methods 9-year-old with $18 \mathrm{~kg}$ bodyweight was complained of abdominal pain, diagnose with peritonitis and planned for laparotomy. Patients underwent with general anesthesia intubation. Drugs for induction include fentanyl $40 \mathrm{mcg}$, ketamine $20 \mathrm{mg}$, and atracurium $10 \mathrm{mg}$ intravenously. Hemodynamics during surgery was stable and after surgery, the patient performed TAP block of Ropivacaine $0.2 \%$ and Clonidine $30 \mathrm{mcg}$ with total volume $5 \mathrm{ml}$ each side for post operative analgesia. Results Patient was operated in COVID-19 operating room which intubation use aerosol box to prevent aerosol contacted

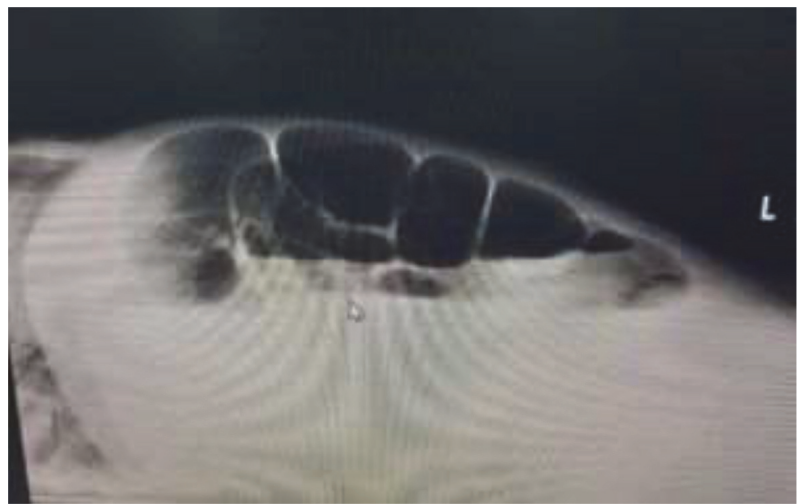

Abstract 226 Figure 1

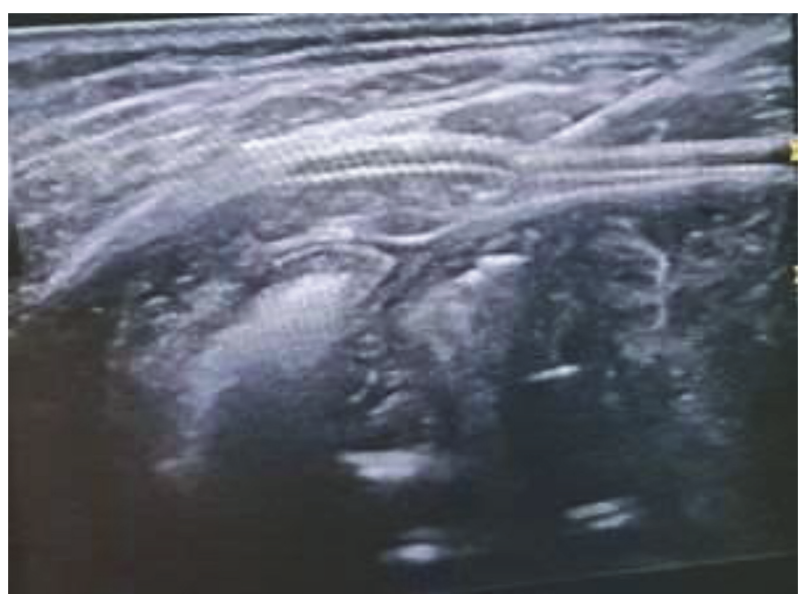

Abstract 226 Figure 2 